\title{
A crucial role of caspase-3 in osteogenic differentiation of bone marrow stromal stem cells
}

\author{
Masako Miura, ${ }^{1}$ Xiao-Dong Chen, ${ }^{1}$ Matthew R. Allen, ${ }^{2}$ Yanming Bi, ${ }^{1}$ Stan Gronthos, ${ }^{3}$ \\ Byoung-Moo Seo, ${ }^{1}$ Saquib Lakhani, ${ }^{4}$ Richard A. Flavell, ${ }^{4}$ Xin-Hua Feng, ${ }^{5}$ \\ Pamela Gehron Robey, ${ }^{1}$ Marian Young, ${ }^{1}$ and Songtao Shi ${ }^{1}$
}

\begin{abstract}
${ }^{1}$ Craniofacial and Skeletal Diseases Branch, National Institute of Dental and Craniofacial Research (NIDCR), NIH, Bethesda, Maryland, USA 2Deptartment of Anatomy and Cell Biology, Indiana University School of Medicine, Indianapolis, Indiana, USA. ${ }^{3}$ Mesenchymal Stem Cell Group, Division of Haematology, Institute of Medical and Veterinary Science, Adelaide, South Australia, Australia. ${ }^{4}$ Section of Immunology, Yale University School of Medicine, New Haven, Connecticut, USA. ${ }^{5}$ Michael E. DeBakey Department of Surgery, Baylor College of Medicine, Houston, Texas, USA.
\end{abstract}

\begin{abstract}
Caspase-3 is a critical enzyme for apoptosis and cell survival. Here we report delayed ossification and decreased bone mineral density in caspase-3-deficient (Casp $3^{-/-}$and $\left.\mathrm{Casp}^{+/-}\right)$mice due to an attenuated osteogenic differentiation of bone marrow stromal stem cells (BMSSCs). The mechanism involved in the impaired differentiation of BMSSCs is due, at least partially, to the overactivated TGF- $\beta / S$ mad2 signaling pathway and the upregulated expressions of $\mathrm{p} 53$ and $\mathrm{p} 21$ along with the downregulated expressions of Cdk2 and Cdc2, and ultimately increased replicative senescence. In addition, the overactivated TGF- $\beta / \mathrm{Smad} 2$ signaling may result in the compromised Runx2/Cbfa1 expression in preosteoblasts. Furthermore, we demonstrate that caspase-3 inhibitor, a potential agent for clinical treatment of human diseases, caused accelerated bone loss in ovariectomized mice, which is also associated with the overactivated TGF- $\beta /$ Smad 2 signaling in BMSSCs. This study demonstrates that caspase-3 is crucial for the differentiation of BMSSCs by influencing TGF- $\beta /$ Smad2 pathway and cell cycle progression.
\end{abstract}

\section{Introduction}

Apoptosis is a process of caspase-mediated cell death and is critical for the normal development and the function of multicellular organisms. The most important impact of apoptosis for the normal function of tissues is to maintain tissue homeostasis by balancing cell proliferation and cell death (1). Caspase- 3 is one of the major activated cysteine proteases that constitute the caspase family and is a pivotal cog in the apoptotic machinery. It cleaves a number of substrates and activates endonucleases, leading to DNA fragmentation, a hallmark of apoptosis (2). Homozygous caspase-3-deficient $\left(\mathrm{Casp}^{-/-}\right)$mice survive for only 3-5 weeks after birth, having neuronal defects and stunted growth, which suggests that caspase- 3 plays a prominent role in normal developmental processes $(3,4)$. Recently, it was found that caspase- 3 activity is required for normal differentiation of $\mathrm{T}$ lymphocytes, erythrocytes, and skeletal muscle cells (5-7), which further indicates that caspase- 3 activity contributes significantly to controlling cell differentiation.

Nonstandard abbreviations used: $\mathrm{BFR}$, bone formation rate; $\mathrm{BMD}$, bone mineral density; BMP, bone morphogenetic protein; BMSSC, bone marrow stromal stem cell;

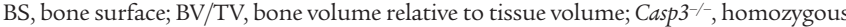
caspase-3-deficient; Casp3Inh, caspase-3 inhibitor; Cdc, cell division cycle; Cdk, cyclin-dependent kinase; CFU-F, CFU fibroblastic; d.p.c., days post coitus; HA/TCP, hydroxyapatite/tricalcium phosphate; MAR, mineral apposition rate; microCT, micro-computed tomography; MS, mineralizing surface; OS, osteoid surface; OVX, ovariectomized; $\mathrm{PQCT}$, peripheral quantitative computed tomography; $\mathrm{p}-\mathrm{Smad}$,

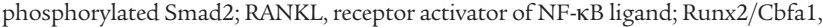
runt-related gene $2 /$ core binding factor $\alpha 1$; Tb.Th, trabecular thickness; TGF- $\beta R I$, TGF- $\beta$ receptor type I; TRAP $-\mathrm{MNC}$, tartrate-resistant acid phosphate-positive multinucleated cell.

Conflict of interest: The authors have declared that no conflict of interest exists.

Citation for this article: J. Clin. Invest. 114:1704-1713 (2004)

doi:10.1172/JCI200420427.
Apoptosis plays an important role during skeletal development and bone remodeling. A recent convergence of clinical and basic research has highlighted a link between osteogenic cell death and osteoporosis $(8,9)$. Recently, it has been suggested that inappropriate apoptosis of osteoblasts/osteocytes accounted for, at least in part, the imbalance of bone remodeling seen in osteoporosis, although elevated activity of osteoclasts has been suggested to be an important factor $(10,11)$. Another study showed decreased osteoblastogenesis with low bone density in a senescence-accelerated mouse model (12). It was also reported that caspase activity was involved in apoptosis of bone marrow stromal cells and osteoblasts $(13,14)$ and may be required for osteogenic differentiation of osteoblastic cell line MC3T3-E1 in vitro (15). Apoptosis of osteoblast may be regulated by a variety of factors such as bone morphogenetic proteins (BMPs) and bone matrix proteins $(16,17)$. Bisphosphonates and calcitonin, commonly used as therapeutic drugs for osteoporosis, can also inhibit apoptosis of osteocyte and osteoblast (18). However, the exact role of caspase- 3 in bone development and skeletal diseases is largely unknown.

The objective of this study was to investigate the functional role of caspase- 3 in the maintenance of normal bone mass in vivo. We found that Casp $3^{-/-}$mice exhibited significant bone defects during early development, while heterozygous caspase-3-deficient $\left(\operatorname{Casp}^{++/-}\right)$ mice displayed a decreased bone mineral density (BMD) with age, a defect consistent with osteoporosis. We further demonstrated that in vivo administration of caspase-3 inhibitor (Casp3Inh) results in an accelerated bone loss especially in ovariectomized (OVX) mice, a widely accepted animal model for postmenopausal osteoporosis due to the deficiency of estrogen $(19,20)$. Finally, Casp3Inhtreated human bone marrow stromal stem cells (BMSSCs) were 
A

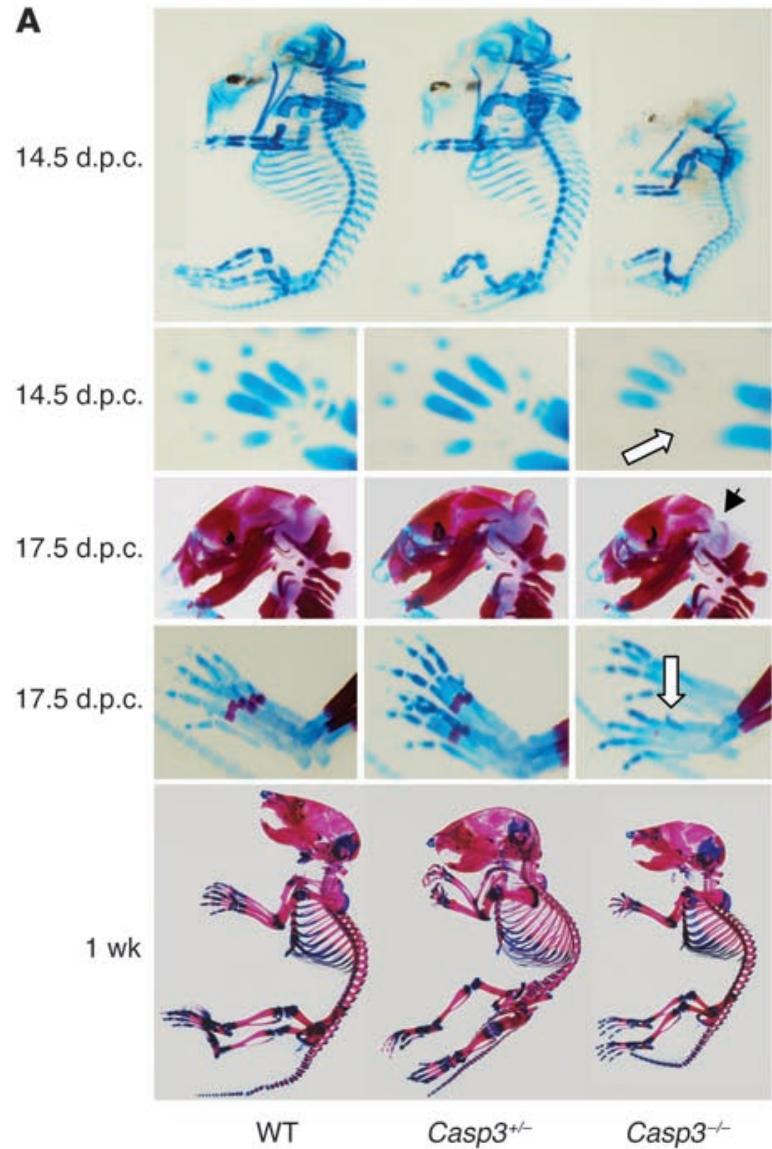

B

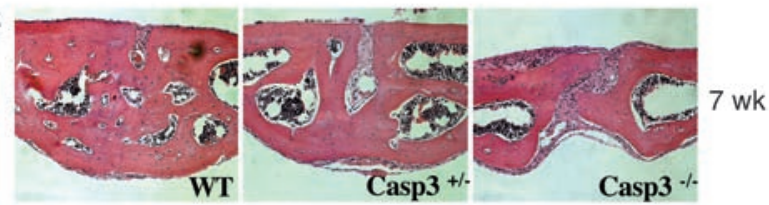

C

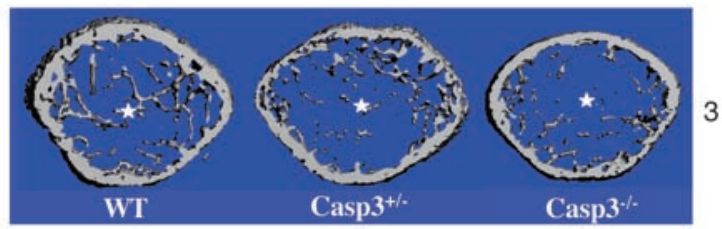

3 wk

D

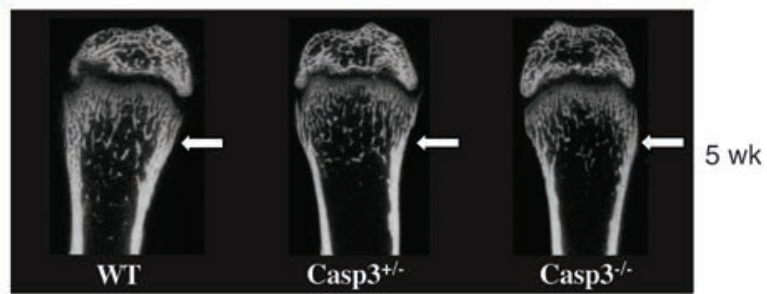

E
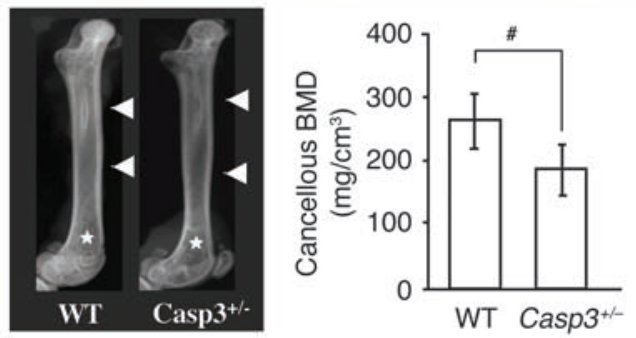

8 wk

\section{Figure 1}

Skeletal defects in caspase-3-deficient mice. (A) Alizarin red and alcian blue staining of mouse skeleton at different stages. Casp3 $3^{-/-}$mice had smaller skeletons and showed delayed development in the skull, metacarpi, phalanges, and the sternum at 14.5 d.p.c. (upper panels). White arrow shows the skeletal developmental delay on metacarpi in Casp3 $3^{-/}$mice. Casp3 $3^{-/}$mice show delayed ossification on interparietal bones (black arrow) and metatarsi (white arrow, middle panels) at 17.5 d.p.c. One-week-old Casp3 $3^{-/}$mice also showed a smaller skeleton (lower panel) compared with WT mice. (B) H\&E staining on the sagittal suture of the anterior frontal bone at 7 weeks. The suture was completely closed in WT mice, while Casp3 $3^{-/}$and Casp3 $^{+/-}$mice showed a completely opened suture and an incomplete closure, respectively. Original magnification, $\times 200$. (C) Trabecular bone structure of the distal femoral metaphysis at 3 weeks by microCT. Both Casp3 $3^{-/-}$and Casp3 $3^{+/-}$mice showed decreased trabecular bone formation (asterisks) compared with WT mice. (D) Coronal sections of the distal femoral metaphysis at 5 weeks by microCT. White arrows show the trabecular bone areas. (E) X-ray images of the femora at 8 weeks (left panel) show differences in bone density between Casp3 $3^{+/-}$and WT mice, including cortical bone (arrowheads) and trabecular bone (asterisks). Cancellous BMD of the distal femora assessed by pQCT (right panel). pQCT also indicated significantly decreased BMD in Casp3 ${ }^{+-}$femora. Error bars represent the mean $\pm \operatorname{SD}\left(n=4\right.$; $\left.{ }^{\#} P<0.05\right)$.

found to have a decreased osteogenic capacity upon in vivo transplantation. This study showed that caspase- 3 activity appears to be a critical component for osteogenic differentiation of BMSSCs associated with replicative senescence of BMSSCs and compromised runt-related gene $2 /$ core binding factor $\alpha 1$ (Runx2/Cbfa1) expression. These data further point to the conclusion that the influence on BMD should be considered upon any in vivo application of Casp3Inhs to the treatment of human diseases, especially in postmenopausal women.

\section{Results}

Skeletal defects in caspase-3-deficient mice. A variety of phenotypes have been reported for Casp $3^{-/-}$mice $(3,4)$. However, there is no description of a skeletal phenotype. In the current study, we found a delayed ossification in $\mathrm{Casp}^{3^{-/}}$mice at 14.5 days post coitus (d.p.c.), which is the initial stage of skeletal development.
The defect of skeletal development was detected in the skull, metacarpi, phalanges, and the sternum at this stage (Figure 1A). The whole skeleton of Casp $3^{-/-}$mice was significantly smaller than that of WT mice. We also confirmed the ossification defects of interparietal bones and metatarsi at 17.5 d.p.c. (Figure $1 \mathrm{~A}$ ) and the delayed ossification of phalanges at 1 week (data not shown). Interestingly, the ossification defects of calvarial bones affected the closure of the coronal suture (Figure 1B). We harvested the boundary area between the frontal bone and the nasal bone from 7 -week-old mice and examined the closure of the sagittal suture on the frontal bone. Casp $3^{-/-}$mice showed either completely opened suture or incomplete closure, depending on the severity of the phenotypes. Casp $3^{+/-}$mice also showed incomplete suture closure, in contrast to WT mice, which exhibited completely closed sutures. However, we cannot exclude the possibility that the enlargement of brain in Casp $3^{-/-}$mice 


$\begin{aligned} & \text { Table 1 } \\ & \text { The 3-dimensional parameters of microCT of femora in 3-week-old mice }\end{aligned}$
$\begin{array}{lllllc} \\ \text { Genotype } & \boldsymbol{n} & \mathbf{B V} / \mathrm{TV}(\%) & \text { Tb.N }\left(\mathbf{m m}^{-1}\right) & \text { Tb.Th }(\mu \mathrm{m}) & \text { Th.Sp }(\mu \mathrm{m}) \\ \text { WT } & 4 & 8.29 \pm 2.63 & 9.68 \pm 1.50 & 34.18 \pm 2.93 & 120.60 \pm 20.22 \\ \text { Casp3 }^{+-} & 4 & 2.36 \pm 0.90^{\mathrm{A}} & 8.09 \pm 0.64 & 29.18 \pm 2.37^{\mathrm{A}} & 138.48 \pm 10.88 \\ \text { Casp3 }^{--} & 4 & 1.96 \pm 0.73^{\mathrm{A}} & 8.01 \pm 0.93 & 25.68 \pm 1.68^{\mathrm{A}} & 143.88 \pm 16.09\end{array}$

Values are shown as mean $\pm \mathrm{SD}$. ${ }^{A} P<0.05$, statistically significant compared with the WT group. Tb.N, trabecular number; Tb.Sp, trabecular separation.

contributes to the observed impaired coronal suture closure. The ossification defects of long bones were also observed. Both micro-computed tomography (microCT) (Figure 1C and Table 1) and bone histomorphometry (Table 2) demonstrated significantly lower bone volume relative to tissue volume (BV/TV), due to a reduced trabecular thickness (Tb.Th), in 3-week-old Casp3 $3^{-/-}$ mice. These parameters were also decreased in $\mathrm{Casp}^{3^{+/-}}$mice, which did not show any significant delay of skeletal maturation at their early developmental stage. Similar tendencies were detected in 5-week-old caspase-3-deficient mice (Figure 1D). $\mathrm{X}$-ray and peripheral quantitative computed tomography (pQCT) analyses confirmed that 2-month-old Casp $3^{+/-}$mice displayed decreased BMD (Figure 1E). The bone defects in Casp3 $3^{-/-}$ mice were obvious at a very early developmental stage. However, the bone phenotype in Casp $3^{+/-}$mice began to appear at approximately 3 weeks of age. The bone phenotypes shown in caspase-3deficient mice support the speculation that normal caspase- 3 activity is critical for maintaining the integrity of bone.

Osteoclast activity is decreased in caspase-3-deficient mice. A balance between bone resorption and bone formation is required for maintaining bone integrity in the remodeling process. Thus, we first examined osteoclast function in vivo and osteoclast induction in vitro using a titanium particle-induced osteolysis assay and a coculture system containing osteoclast progenitors derived from spleen and preosteoblasts derived from calvariae, respectively. Osteoclast function was significantly decreased in Casp $3^{-/-}$mice compared with WT mice (Figure 2A), which indicated that osteoclast-mediated bone resorption was not a major cause of observed bone defects for Casp $3^{-/-}$mice. When osteoclast progenitors from WT mice were cocultured with preosteoblasts from Casp $3^{-/-}$and $\mathrm{Casp}^{+/-}$mice, the number of tartrate-resistant acid phosphate-positive multinucleated cells (TRAP ${ }^{+}$-MNCs) was significantly decreased (Figure 2B). Collectively, these data suggest that impaired osteoclast activity in caspase-3-deficient mice might be associated with dysfunctional preosteoblastic cells. The downregulated expression of receptor activator of NF- $\mathrm{KB}$ ligand (RANKL) in Casp $3^{-/-}$and Casp $3^{+/-}$preosteoblasts also suggested that impaired osteoclastogenesis in caspase-3-deficient mice might be caused by impaired preosteoblasts (Figure 2C). Therefore, we focused on investigating cell proliferation and osteogenic differentiation of BMSSCs derived from caspase-3-deficient mice.

Proliferation and differentiation of BMSSCs are compromised in caspase-3-deficient mice. CFU fibroblastic (CFU-F) assay was used to determine the number of stromal progenitors. The number of colonies generated from 3-week-old Casp3 $3^{-/-}$and Casp $3^{+/-}$mice was significantly lower than that of the age-matched WT mice (Figure 3A). The number of proliferating BMSSCs in $\mathrm{Casp}^{3^{-/-}}$and $\mathrm{Casp}^{+/-}$mice was significantly decreased compared with WT BMSSCs as assessed by the BrdU incorporation assay (Figure 3B). These observations implied that the reduction of CFU-F number in caspase-3deficient mice might be associated with a quantitative reduction in osteogenic cells. In the mean time, when cultured under the osteogenic inductive condition, Casp3 $3^{--}$ and Casp $3^{+/-}$BMSSCs accumulated less calcium compared with WT BMSSCs (Figure 3C). In vivo dynamic histomorphometric analysis also showed that distal femoral cancellous bone of caspase-3-deficient mice exhibited reduced osteoid surface/bone surface (OS/BS) percentage, mineralizing surface/BS (MS/BS) percentage, mineral apposition rate (MAR), and bone formation rate (BFR) compared with WT mice (Table 2). Additionally, the osteogenic capacity of BMSSCs was examined by in vivo transplantation into immunocompromised mice using hydroxyapatite/tricalcium phosphate (HA/TCP) as a carrier vehicle. Casp3 $3^{-1-}$ BMSSCs generated significantly less bone at 8 weeks after transplantation compared with WT BMSSCs (Figure 3D).

Overactivation of $T G F-\beta / S m a d 2$ signaling accelerates replicative senescence by inhibiting cell cycle progression in caspase-3-deficient BMSSCs. We treated BMSSCs with TGF- $\beta$ and examined replicative senescence and apoptosis, respectively. We observed that TGF- $\beta$ induced an accelerated replicative senescence in $\mathrm{Casp}^{3^{-/-}}$and Casp $3^{+/-}$BMSSCs as assessed by $\beta$-gal staining (Figure $4 \mathrm{~A}$ ). In contrast, BMP- 2 treatment did not induce an increased senescence in Casp3 $3^{-/-}$or Casp $3^{+-}$ BMSSCs (data not shown), which indicates there was a TGF- $\beta$ specificity in the process of senescence. Annexin V staining showed that Casp $^{3^{-/}}$BMSSCs exhibited a decreased level of apoptosis in the presence of TGF- $\beta$ (Figure $4 \mathrm{~B}$ ). Even in the absence of TGF- $\beta$ treatment, both Casp $3^{-/-}$and $\mathrm{Casp}^{+/-}$BMSSCs showed a decreased population doubling along with morphological features of senescence such as enlarged cells and abnormal nuclei (Figure 4C). To elucidate the mechanism whereby TGF- $\beta$ accelerates senescence in Casp3 $3^{-1-}$ BMSSCs, we examined the involvement of the TGF- $\beta$-mediated signaling proteins and cell cycle-related proteins. Casp3 $3^{-1-}$ BMSSCs showed elevated expression of TGF- $\beta$ receptor type I (TGF- $\beta$ RI) and Smad 2 under the regular culture condition. After TGF- $\beta$ treatment, the expression of Smad2 and phosphorylated Smad2 (p-Smad2) was upregulated compared with that of WT BMSSCs (Figure 4D), which indicates that TGF- $\beta / S \operatorname{Smad} 2$ pathway was overactivated in Casp3 $3^{-/-}$BMSSCs. In contrast, TGF- $\beta$ RII and Smad3 were not upregulated in Casp3 $3^{-/}$BMSSCs (Figure 4D). Moreover, the expressions of cyclin-dependent kinase $2(\mathrm{Cdk} 2)$ and cell division cycle 2 (Cdc2) were downregulated in $\mathrm{Casp}^{-1-}$ BMSSCs, while the

\section{Table 2}

Distal femur cancellous bone histomorphometry

$\begin{array}{lllrcc}\text { Genotype } & \text { BV/TV (\%) } & \text { OS/BS (\%) } & \text { MAR }(\mu \mathrm{m} / \mathbf{d}) & \text { MS } / \mathbf{B S}(\%) & \text { BFR }\left(\mu \mathbf{m}^{3} / \mu \mathbf{m}^{2} / \mathbf{y r}\right) \\ \text { WT } & 9.54 \pm 1.28 & 2.48 \pm 0.43 & 1.41 \pm 0.44 & 15.9 \pm 2.9 & 106 \pm 62 \\ \text { Casp3 }^{+/} & 5.12 \pm 1.50 & 2.58 \pm 0.45 & 0.85 \pm 0.23 & 8.5 \pm 1.2^{\mathrm{A}} & 34 \pm 12 \\ \text { Casp3 }^{--} & 4.58 \pm 0.86^{\mathrm{A}} & 0.32 \pm 0.32^{\mathrm{A}} & 0.67 \pm 0.24 & 6.61 \pm 1.6^{\mathrm{A}} & 27 \pm 11\end{array}$

The numbers of analyzed mice were 5 (WT) and $3\left(\mathrm{Casp}^{+/-}\right.$and $\left.\mathrm{Casp}^{-/-}\right)$for measuring BV/TV and OS/BS and 10 (WT and Casp3 $\left.^{--}\right)$and $14\left(\right.$ Casp3 $\left.^{+/-}\right)$for MAR, MS/BS, and BFR, respectively. Values are shown as mean $\pm S E .{ }^{A} P<0.05$, statistically significant compared with the WT group. 
A
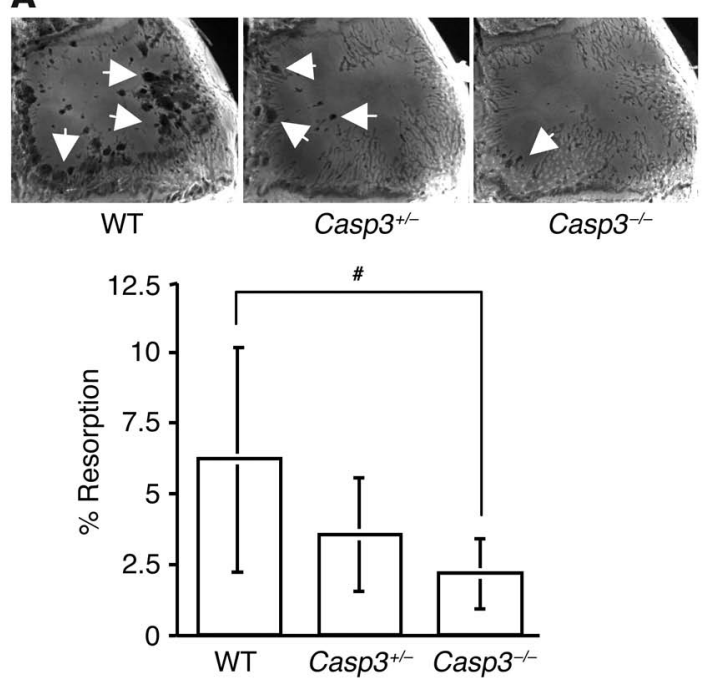

Figure 2

Osteoclast function in caspase-3-deficient mice. (A) Representative images of titanium-induced resorption in the calvarial bones (white arrows) of WT, Casp3 $3^{+-}$, and $\mathrm{Casp}^{-/-}$mice. There was a significant decrease in bone resorption in Casp3 $3^{-/-}$mice compared with WT mice (WT, $n=12$; Casp3 $3^{+-}, n=12$; Casp3 $3^{--}, n=8$; $\# P<0.05$ ). (B) The number of induced TRAP+-MNCs. Spleen cells from WT mice were cocultured with preosteoblasts from WT, Casp3 $3^{+-}$, and Casp3-/- mice. The number of TRAP+-MNCs was significantly decreased in $\mathrm{Casp}^{-/-}$and $\mathrm{Casp}^{+/-}$preosteoblasts compared with WT (+/+) preosteoblasts

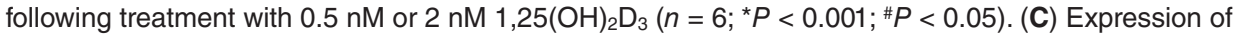
RANKL. RANKL was downregulated in $\mathrm{Casp}^{-/-}$preosteoblasts compared with WT preosteoblasts by Western blot analysis. The expression of HSP90 is shown as a control for each protein loading.
B

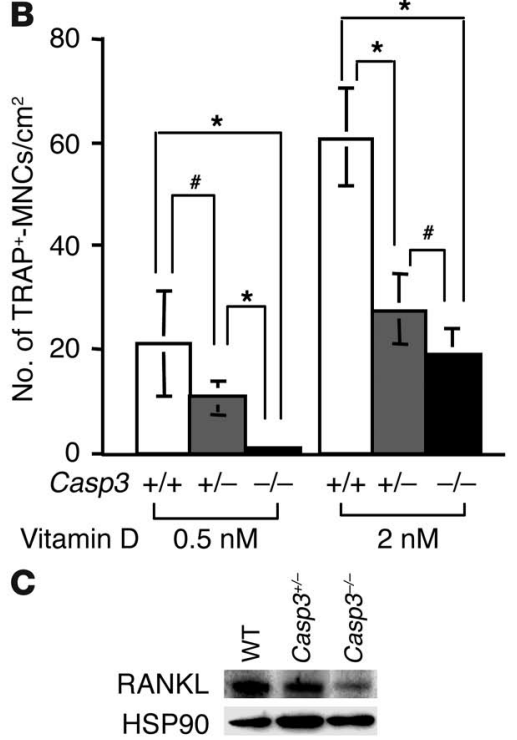

pared with treatment with vehicle (DMSO) (Figure 6A). As observed in the caspase-3-deficient mice, there was no increased bone resorption in Z-DEVD-FMK-treated OVX (Casp3Inh-OVX) mice compared with DMSO-treated OVX (DMSOOVX) mice (data not shown), which suggests that the osteoclast function was not a major factor for the decreased BMD observed in Casp3Inh-OVX mice. Even in shamoperated mice, Z-DEVD-FMK treatment induced a decreased BMD (Figure 6A). As expected, BMSSCs derived from Casp3Inh-OVX mice showed significant increase in expressions of TGF- $\beta$ RI, Smad2, and $\mathrm{p}-\mathrm{Smad} 2$ compared with those from DMSO-OVX mice (Figure 6B). Upregulated expression of Smad2 was also shown in BMSSCs from Z-DEVD-FMK-treated shamoperated (Casp3Inh-sham) mice. Interestingly, BMSSCs derived from Casp3Inh-OVX mice showed a detectable level of $\mathrm{p}-\mathrm{Smad} 2$ in the absence of TGF- $\beta$ treatment. In contrast, the expression of Smad3 in BMSSCs was not significantly increased in any group.

expression of their counterparts, p21 and p53, was upregulated (Figure 4D), which may be one of the mechanisms leading to cell cycle arrest and eventual replicative senescence. We failed to detect p21 expression in WT BMSSCs until 3 days after TGF- $\beta$ treatment (Figure 4D), although p21 expression became detectable in WT BMSSCs after 7 days of TGF- $\beta$ treatment (data not shown). Interestingly, even in the absence of TGF- $\beta$ treatment, upregulated TGF- $\beta$ RI, Smad2, p21, and p53 along with downregulated Cdc2 were found in Casp $3^{-1-}$ BMSSCs (Figure 4D). These data implied that caspase-3 deficiency caused overactivation of the TGF- $\beta$ signaling pathway and attenuation of the cell cycle in BMSSCs.

We further examined the involvement of the osteogenic master protein, Runx $2 / \mathrm{Cbfa} 1$, in osteoprogenitors derived from mouse calvariae. We found a significant decrease in expression of Runx2/ Cbfa1 in $\mathrm{Casp}^{3^{-/-}}$and Casp $3^{+/-}$preosteoblasts (Figure 5). Upon TGF- $\beta$ treatment, Runx $2 / C b f a 1$ expression was significantly elevated in $\mathrm{Casp}^{++-} \mathrm{BMSSCs}^{2} 4$ hours after treatment. With continuous treatment for 7 days, there was a downregulated expression of Runx $2 / \mathrm{Cbfa} 1$ in WT and $\mathrm{Casp}^{+/-}$BMSSCs. Interestingly, TGF- $\beta$ was not able to alter Runx2/Cbfa1 expression in Casp $3^{-/-}$BMSSCs. In contrast, BMP- 2 treatment did not induce any differential Runx2/Cbfa1 expression in preosteoblasts from caspase-3-deficient mice (data not shown), which suggests that TGF- $\beta$ specifically altered Runx2/Cbfa1 expression.

Casp3Inh accelerates bone loss in OVX mice. The Casp3Inh Z-DEVDFMK was administered to mice to examine whether inhibition of caspase- 3 activity could achieve similar results as observed in caspase-3-deficient mice. We found that BMD in OVX mice was significantly decreased after treatment with Z-DEVD-FMK com-
Casp3Inh suppresses osteogenic differentiation of human BMSSCs. To pursue the potential influence of caspase-3 on human cells, Z-DEVD-FMK was used to treat human BMSSCs, and the osteogenic potential of BMSSCs was assessed. Z-DEVD-FMK was capable of inhibiting caspase- 3 activity in cultured human BMSSCs (Figure 6C). Moreover, we found that Z-DEVD-FMK was able to suppress the calcium accumulation of human BMSSCs in vitro (Figure 6D) and inhibited bone formation of human BMSSCs in vivo (Figure 6E). These data confirmed that caspase-3 plays a pivotal role in the differentiation of human BMSSCs.

\section{Discussion}

Here we provide evidence to support the notion that caspase- 3 is critical for osteogenic differentiation of BMSSCs using 3 different models: caspase-3-deficient mice, Casp3Inh-treated mice, and Casp3Inh-treated human BMSSCs. As shown in the analysis of caspase-3-deficient mice, complete caspase-3 deficiency (Casp $3^{-/-}$) caused bone defects at early developmental stages, while partial caspase-3 deficiency (Casp $\left.3^{+-}\right)$led to an age-dependent decrease in BMD. The decreased BMD in OVX mice was exacerbated by the inhibition of caspase-3 activity using Casp3Inh. These findings indicate that caspase- 3 plays a critical role in vivo in both processes of bone development and bone remodeling.

Accumulated evidence suggests that apoptosis of osteogenic cells is involved in bone defects. We found that BMSSCs derived from caspase-3-deficient mice, including Casp $3^{-/-}$and Casp $3^{+/-}$mice, demonstrated 2 distinct features; decreased proliferation capability and impaired osteogenic differentiation. The former was indicated by CFU-F assay, BrdU incorporation assay, and continuous 
A
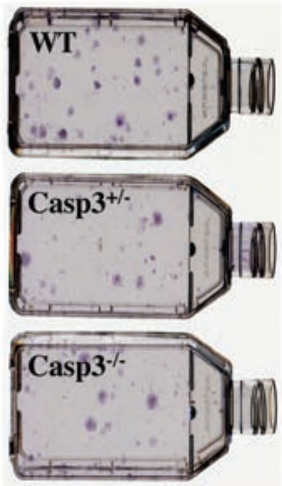

C

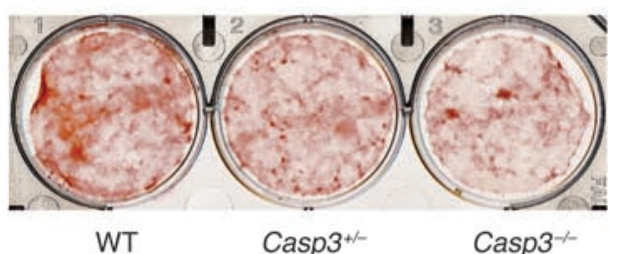

B

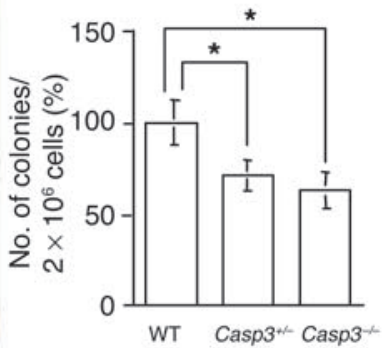

Casp3 $^{+1-}$

D
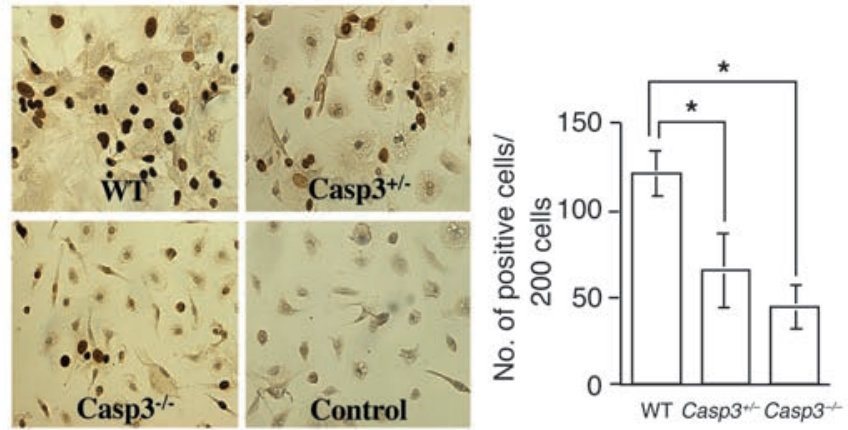

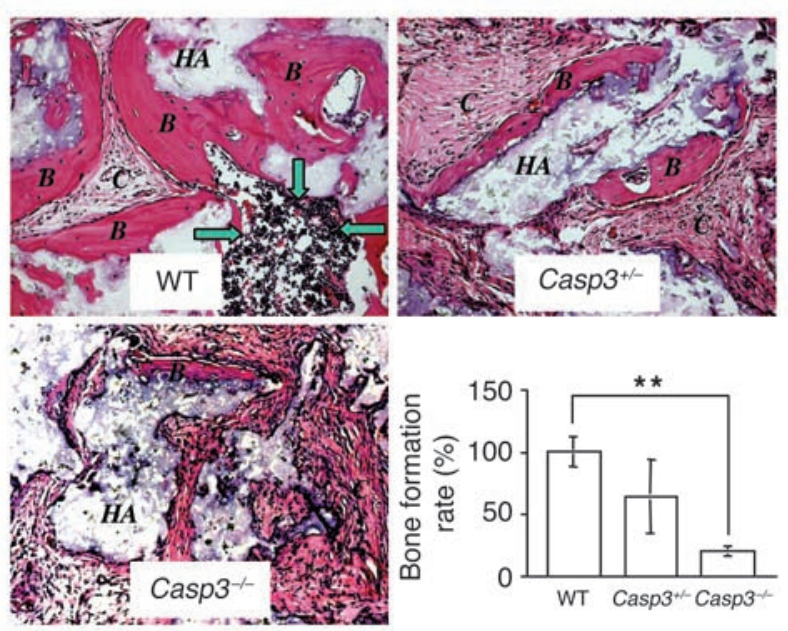

Figure 3

Proliferation and osteogenic differentiation of BMSSCs in caspase-3-deficient mice. (A) Appearance of CFU-F derived from WT, Casp3 $3^{+/}$, and Casp $^{-/-}$mice (left). There was a significant difference in the number of colonies between caspase-3-deficient mice (Casp3 $3^{-/-}$and Casp3 $3^{+/}$) and WT mice (right). Error bars represent the mean $\pm \mathrm{SD}\left(n=10 ;{ }^{*} P<0.001\right)$. (B) BrdU incorporation of BMSSCs. The proliferation rate of cultured BMSSCs was assessed by BrdU incorporation assay for 24 hours. Representative pictures are shown at left. Original magnification, $\times 400$. The number of BrdU-positive cells is indicated as a percentage of the total number of counted BMSSCs and averaged from 5 replicated cultures. Error bars represent mean $\pm \mathrm{SD}\left(n=5 ;{ }^{*} P<0.001\right)$. (C) Alizarin red staining of BMSSCs cultured under the osteogenic inductive condition.

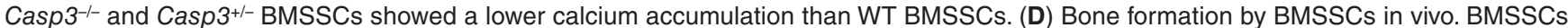
were transplanted into immunocompromised mice with HA/TCP $(\mathrm{HA})$. Bone formation assessed by H\&E staining was decreased in Casp $3^{-1-}$ and $\mathrm{Casp}^{+/-}$transplants compared with WT transplants. B, bone; C, connective tissue; green arrows, hematopoeitic marrow elements. Original magnification, $\times 200$. The BFR was calculated as the percentage of newly formed bone area per total area of transplant at the representative

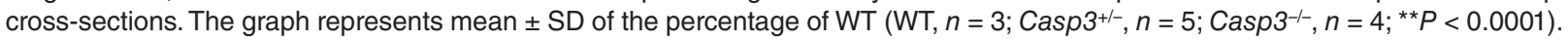

passage assay. To show the impaired osteogenic differentiation, we performed alizarin red staining on BMSSCs cultured under the osteogenic inductive condition and showed a decreased calcium accumulation in caspase-3-deficient BMSSCs. In addition, we showed significantly decreased OS, MS, MAR, and BFR in Casp $3^{-/-}$mice using bone histomorphometric analysis. Moreover, bone formation capacity was attenuated in Casp $3^{--}$BMSSCs using in vivo transplantation assay. In the meantime, osteoclast activity and osteoclast induction were not increased in caspase-3-deficient mice, which indicates that increased bone resorption was not a potential cause for the decreased BMD. Taken together, these findings suggest that the dysfunction of BMSSCs was a major factor for the bone defects in $\mathrm{Casp}^{-/-}$mice.

At the molecular and cellular levels, Casp $3^{-/-}$BMSSCs exhibited increased replicative senescence along with simultaneously decreased apoptosis as shown by $\beta$-gal staining, Annexin V stain- ing, and population doubling. Senescence and apoptosis are the events that occurred at the $G_{0} / G_{1}$ phase of the cell cycle. p21 was demonstrated to be a caspase- 3 substrate in human hepatoma cell line (21), and was further identified as playing an important role in switching cellular apoptosis to senescence in response to anticancer drug treatment when apoptosis was blocked by a caspase inhibitor (22). p21 activity, which is upregulated by p53, is able to inhibit the expression of Cdks. This evidence may provide an explanation for why upregulated $\mathrm{p} 21 / \mathrm{p} 53$ and downregulated $\mathrm{Cdk} 2 / \mathrm{Cdc} 2$ were found in Casp $3^{-/-}$BMSSCs.

TGF- $\beta$ can exert diverse effects depending on cell types (23-25). In bone-forming cells, TGF- $\beta$ has been demonstrated to induce cell proliferation and inhibit osteogenic differentiation by repression of Smad3-mediated Runx2/Cbfa1 expression (26). However, the way in which Smad2 and Smad3 become involved in response to TGF- $\beta$ varies according to the cell types. In this study, we found 
A

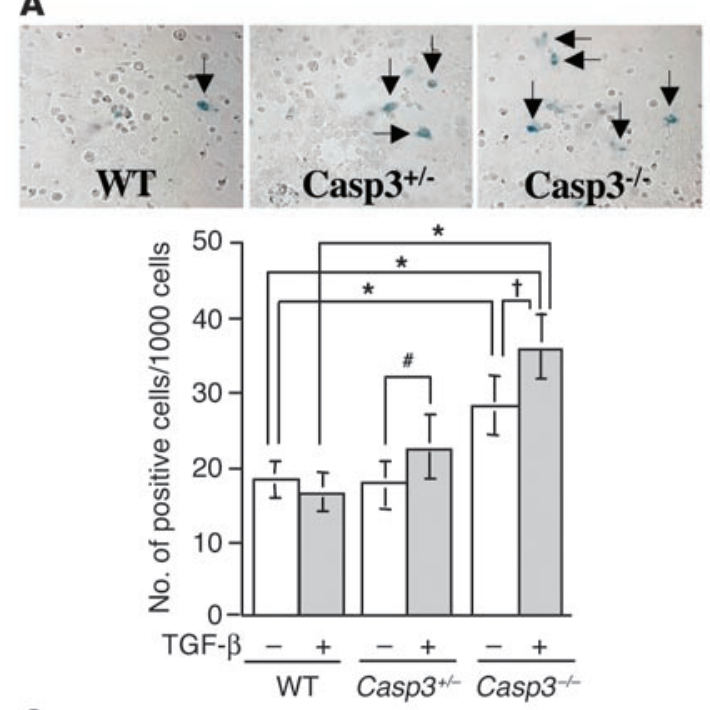

C
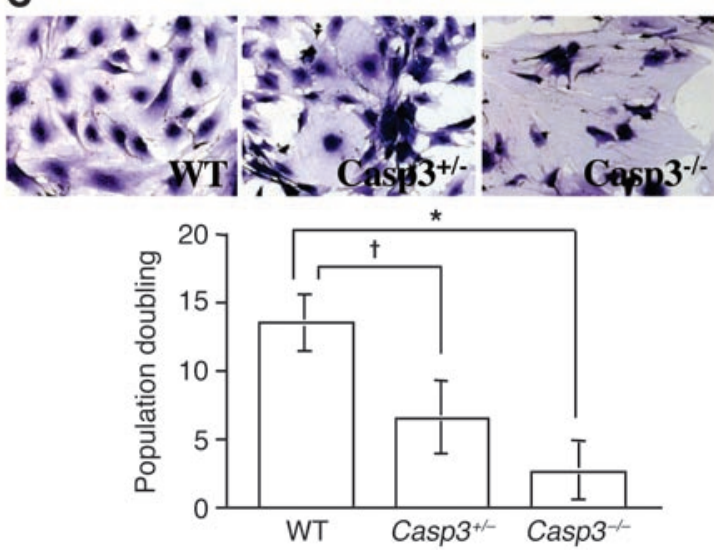

B

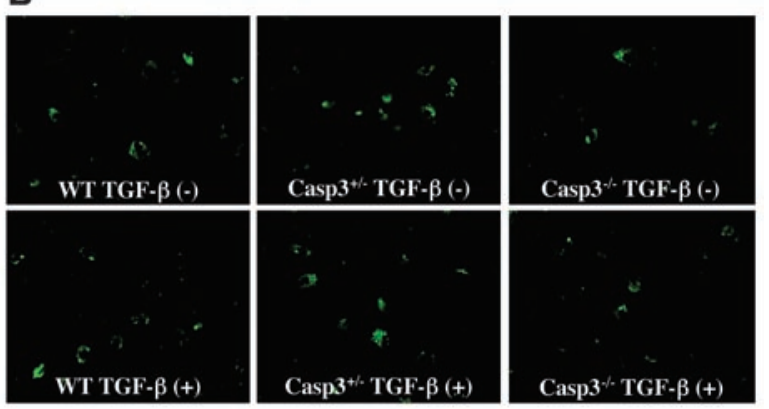

D

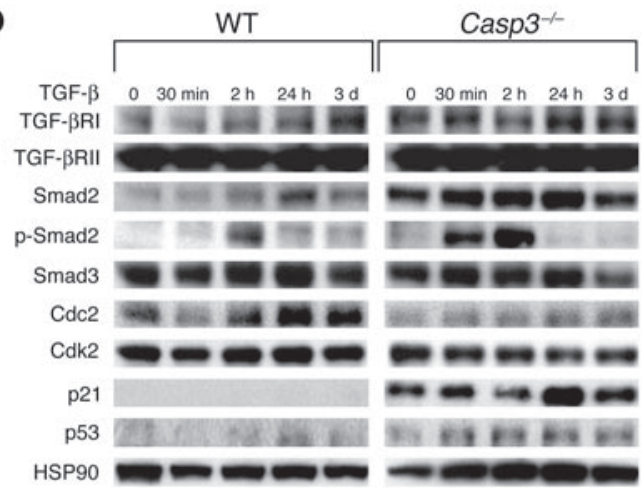

Figure 4

TGF- $\beta$-associated replicative senescence of caspase-3-deficient BMSSCs. (A) Replicative senescence assessed by $\beta$-gal staining. Representative pictures of $\beta$-gal-positive cells induced by TGF- $\beta$ are shown in the upper panel (arrows). Original magnification, $\times 200$. Replicative senescence was increased in Casp3 ${ }^{-1-}$ BMSSCs compared with WT BMSSCs (lower panel; $n=6 ;{ }^{*} P<0.001$ ). TGF- $\beta$ accelerated senescence in caspase-3-deficient BMSSCs ( ${ }^{\dagger} P<0.01 ; \# P<0.05$ ) but not in WT mice. (B) Annexin V staining of BMSSCs. The number of annexin V-positive cells was found to be similar among each genotype under the regular culture condition (-, upper panels). However, TGF- $\beta$ treatment reduced the number of annexin $\mathrm{V}-$ positive cells in Casp3 ${ }^{--}$BMSSCs (+, lower panels). Annexin V-positive cells appear in green. Original magnification, $\times 200$. (C) Population doubling of BMSSCs. BMSSCs were continuously passaged at the same cell density after confluency. Fifty days after the culture was started, Casp3 $3^{-/-}$BMSSCs stopped proliferating and showed enlarged cell body and nuclei, although WT BMSSCs continued proliferating (upper panels). Caspase-3-deficient BMSSCs showed decreased population doubling (lower panel) $\left(n=6 ;{ }^{*} P<0.001 ;{ }^{\dagger} P<0.01\right)$. (D) Western blot analysis of BMSSCs. Casp3 ${ }^{-/-} \mathrm{BMSSCs}$ showed upregulated expression of TGF- $\beta$ RI, Smad2, p21, and p53 along with downregulated expression of Cdc2 compared with WT. After TGF- $\beta$ treatment, expression of TGF- $\beta$ RI, Smad2, p-Smad2, p21, and p53 was further upregulated accompanying with downregulated expression of Cdk2 and Cdc2. Smad3 and TGF- $\beta$ RII expressions were not changed in Casp3 $3^{-/-}$BMSSCs even with TGF- $\beta$ treatment. Ten micrograms of protein was applied to each lane, and HSP90 was used as an additional control for protein loading.

that Smad2 contributed significantly to upregulation of TGF- $\beta$ signaling in $\mathrm{Casp}^{-/-}$BMSSCs. BMSSCs might act to compensate replicative senescence by activating the TGF- $\beta / \operatorname{Smad} 2$ pathway to induce cell proliferation under the situation of caspase- 3 deficiency. TGF- $\beta$-mediated $G_{0} / G_{1}$ phase events have been well studied in epithelial cells in which TGF- $\beta$ may inhibit Cdc 2 and Cdk4 synthesis $(27,28)$, suppress Cdk2 expression $(29,30)$, and induce expression of p21 and p27 $(31,32)$. Upregulated TGF- $\beta /$ Smad 2 signaling pathway may interfere with the regulation of cell cyclerelated molecules such as $\mathrm{Cdc} 2, \mathrm{Cdk} 2, \mathrm{p} 21$, and p53, leading to defects of proliferation and differentiation in caspase-3-deficient
BMSSCs. Additionally, the upregulated TGF- $\beta / \operatorname{Smad} 2$ pathway appeared to alter the expression pattern of Runx $2 / \mathrm{Cbfa} 1$ in preosteoblasts, which may indicate a more direct negative influence on the osteogenic differentiation of BMSSCs. Therefore, it is reasonable to speculate that the overregulated TGF- $\beta / \operatorname{Smad} 2$ signaling pathway, along with upregulated expression of p21/p53 and downregulated expression of $\mathrm{Cdk} 2 / \mathrm{Cdc} 2$ in BMSSCs, resulted in cell cycle arrest and replicative senescence, leading to an impaired osteogenesis in $\mathrm{Casp}^{3^{-/}}$mice.

Abnormalities in apoptosis contribute to a variety of diseases, including cancers (33), autoimmune diseases (34), and degen- 


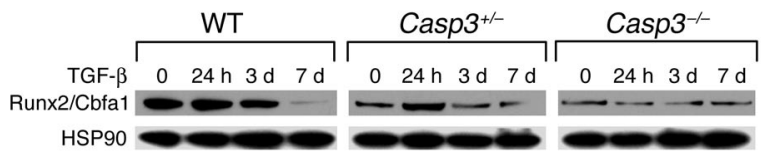

Figure 5

Runx2/Cbfa1 expression in preosteoblasts by Western blot analysis. Caspase-3-deficient $\left(\mathrm{Casp}^{-/-}\right.$and $\mathrm{Casp}^{+/-}$) preosteoblasts showed decreased Runx2/Cbfa1 expression and altered expression pattern of Runx2/Cbfa1 in response to TGF- $\beta$ treatment when compared with WT preosteoblasts.

erative disorders $(35,36)$. Inhibition of caspase activities has been considered to be a novel therapeutic strategy for a variety of apoptosis-related diseases involving nervous system defects (37), retinal degeneration (38), liver injury (39), stunned myocardium (40), and sepsis (41), by reducing apoptosis and thereby improving organ function (42). Z-DEVD-FMK is a widely used Casp3Inh, but the specificity of its caspase-3-inhibiting function should be assessed carefully, since it may influence the activities of other caspases. In this study, we treated mice with Z-DEVD-FMK and found that inhibition of caspase- 3 activity influenced BMD through upregulated TGF- $\beta / \operatorname{Smad} 2$ signaling pathway in BMSSCs, confirming that a balanced caspase- 3 activity may be a prerequisite for normal osteogenic differentiation of BMSSCs to maintain bone integrity. More significant influence was observed in bones of OVX mice than that of sham-operated mice. This finding suggests that Casp3Inh may strongly affect the cells with high turnover rates, such as osteoprogenitors in postmenopausal women. We also confirmed that Z-DEVD-FMK blocks caspase- 3 activity and inhibits osteogenic differentiation in human BMSSCs. These data suggest the necessity for careful consideration of any application of Casp3Inhs, especially in postmenopausal women.
A



C

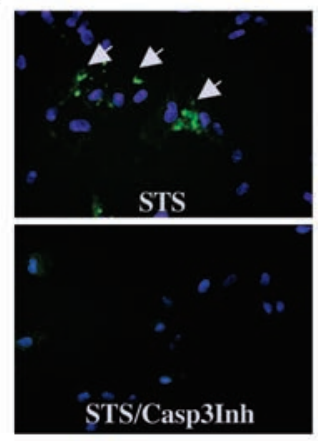

D

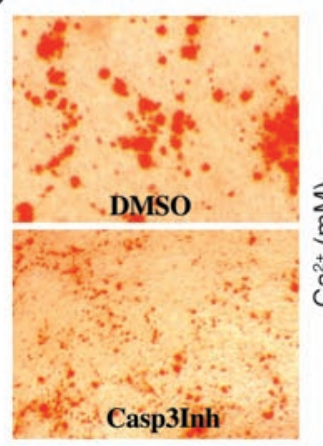

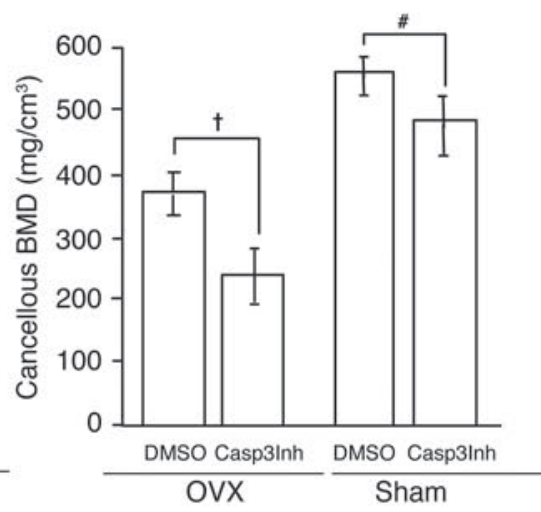

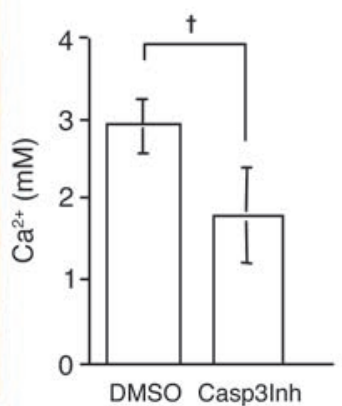

B

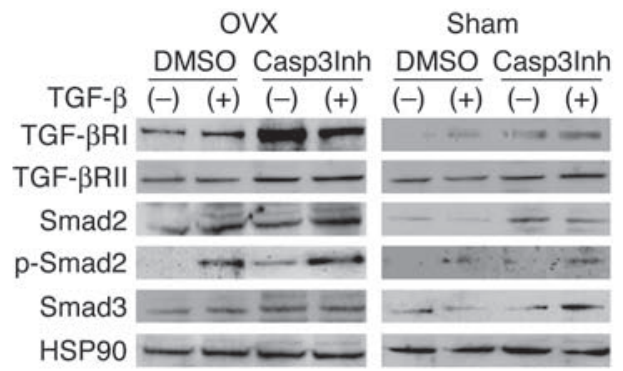

E

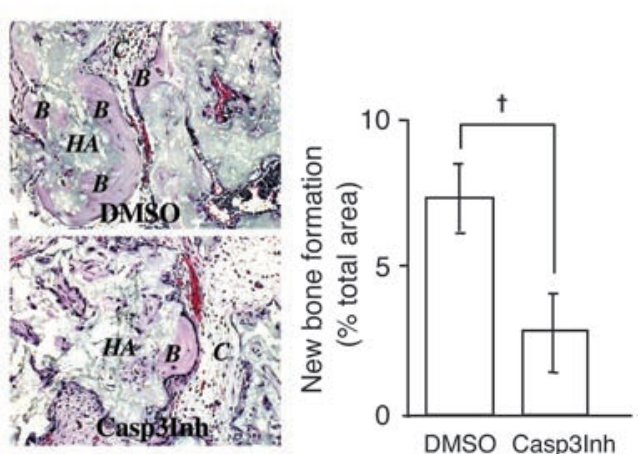

Figure 6

Administration of Casp3Inh to mice and cultured human BMSSCs. (A) BMD in Casp3Inh-treated mice. X-ray images show bone density differences in femora between Casp3Inh-treated mice and DMSO-treated mice after both OVX and sham operation (left). Arrows and asterisks represent cortical and trabecular bone area in femora, respectively. PQCT analysis also showed decreased cancellous BMD of distal femora in Casp3Inhtreated mice, especially after OVX (right). Error bars represent the mean $\pm \mathrm{SD}\left(n=4 ;{ }^{\dagger} P<0.01 ; \# P<0.05\right)$. (B) Western blot analysis of human BMSSCs. Casp3Inh-treated BMSSCs showed upregulated expression of TGF- $\beta$ RI and TGF- $\beta$ RII compared with DMSO-treated BMSSCs, especially after OVX. After TGF- $\beta$ treatment, the expression of Smad2 and p-Smad2 was increased both in Casp3Inh-OVX and DMSO-OVX BMSSCs. Even without TGF- $\beta$ treatment, $p$-Smad2 was detectable in BMSSCs from Casp3Inh-OVX mice. (C) Caspase-3 activity in human BMSSCs. After BMSSCs were treated with staurosporin (STS), caspase-3 activity was detected (upper panel, green staining, arrows). Caspase-3 activity was not detectable with pretreatment with Casp3Inh (lower panel). Blue color represents nuclei staining with DAPI. Original magnification, $\times 200$. (D) Alizarin red staining of human BMSSCs. BMSSCs were treated with Casp3Inh or DMSO for 24 hours and then cultured under the osteogenic inductive condition. Calcium accumulation was decreased in Casp3Inh-treated BMSSCs (left). Matrix calcium levels released by acid treatment were measured (right). Error bars represent the mean $\pm \mathrm{SD}\left(n=4 ;{ }^{\dagger} P<0.01\right)$. (E) Bone formation by human BMSSCs in vivo. Bone formation assessed by H\&E staining was decreased in the Casp3Inh-treated BMSSC transplant (left). Original magnification, $\times 200$. The BFR was calculated as the percentage of newly formed bone area per total area of transplant at the representative cross-sections. Error bars represent the mean \pm SD (right panel; $n=5 ; \uparrow P<0.01$ ). 
In this study, we found that caspase-3 activity was required for the functional differentiation of BMSSCs. Increased replicative senescence and altered expression of Runx $2 / \mathrm{Cbfa} 1$ through upregulated TGF- $\beta /$ Smad 2 signaling may contribute to the impaired osteogenic differentiation of BMSSCs. In conclusion, caspase-3 plays a crucial role in maintaining both bone development and metabolism. The influence of Casp3Inhs on BMD should be considered for any further clinical application.

\section{Methods}

Animals. The generation of Casp $3^{-/-}$mice has been described previously (3). For dynamic bone histomorphometric analysis, calcein $(20 \mathrm{mg} / \mathrm{kg}$ body weight; Sigma-Aldrich) was injected intraperitoneally into 3-weekold mice twice (at an interval of 3 days) to label actively forming bone surfaces. Casp3Inh (Z-DEVD-FMK, $10 \mathrm{mM}$; BioVision Inc.) dissolved in DMSO was injected to 12 -week-old C3H mice (The Jackson Laboratory) intraperitoneally at a dosage of $0.4 \mu \mathrm{l} / \mathrm{g}$ body weight twice a week for 2 weeks starting 2 days after ovariectomy. An equal volume of DMSO was injected as a control. The mice were sacrificed 4 weeks after ovariectomy. All animal experiments were approved by the NIDCR Animal Care and Use Committee at NIH.

Mouse BMSSCs culture. Mouse bone marrow cells $\left(1-2 \times 10^{7}\right)$ harvested from long bones were seeded into 100-mm culture dishes, incubated for 3 hours at $37^{\circ} \mathrm{C}$ to allow attachment of adherent cells, and then rinsed twice with PBS to remove the nonadherent cells. Bone marrow cells $\left(1-2 \times 10^{7}\right)$ from long bones of guinea pigs were then added into each dish as feeder cells. To prevent proliferation in culture, the feeder cells were $\gamma$-irradiated (Caesium-137) with 6,000 cGy by a Gammacell-1000 Irradiator (Atomic Energy of Canada Ltd.). BMSSCs formed adherent colonies after 12-15 days of culture. Primary cultures were passed to disperse the colony-forming cells (passage 1). The cells at passage 1 at approximately $70 \%$ confluency were utilized for the experiments. Culture medium consisted of $\alpha$-MEM (Gibco BRL; Invitrogen Corp.), 20\% fetal bovine serum (FBS; EquitechBio Inc.), $2 \mathrm{mM}$ L-glutamine, a combination of $100 \mathrm{U} / \mathrm{ml}$ penicillin and $100 \mu \mathrm{g} / \mathrm{ml}$ streptomycin (Biofluids Inc.), and $55 \mu \mathrm{M} 2$-mercaptoethanol (Gibco BRL; Invitrogen Corp.). For the osteogenic induction in vitro, $2 \mathrm{mM} \beta$-glycerophosphate (Sigma-Aldrich), $100 \mu \mathrm{M}$ L-ascorbic acid 2-phosphate (Wako Pure Chemical Industries Ltd.), and $10 \mathrm{nM}$ dexamethasone (Sigma-Aldrich) were added to the culture for 6 weeks. For experiments of TGF- $\beta$ (R\&D Systems Inc.) treatment, the cells were serum-starved with $\alpha$-MEM supplemented with $2 \%$ FBS for 16 hours prior to the treatment with $2 \mathrm{ng} / \mathrm{ml} \mathrm{TGF}-\beta$. In the case of treatment for 3 days, the cells were incubated with TGF- $\beta$ in regular culture medium.

Human BMSSCs culture. Human bone marrow aspirates from healthy human adult volunteers (20-35 years of age) were purchased from Poietic Technologies Inc. and cultured as reported previously (43). Single-cell suspensions $\left(0.01-1 \times 10^{5} /\right.$ well $)$ of BMSSCs were cultured in 6-well plates (Corning Costar Co.) with $\alpha$-MEM supplemented with 15\% FBS, $100 \mu \mathrm{M}$ L-ascorbic acid 2-phosphate, $2 \mathrm{mM}$ L-glutamine, and a combination of $100 \mathrm{U} / \mathrm{ml}$ penicillin and $100 \mu \mathrm{g} / \mathrm{ml}$ streptomycin. After Z-DEVD-FMK was added at $2 \mu \mathrm{M}$ for 24 hours, BMSSCs were treated with TGF- $\beta$ for 24 hours and harvested for Western blot analysis; however, for the purpose of detecting expression of $\mathrm{p}$-Smad2, BMSSCs were harvested after TGF- $\beta$ treatment for 30 minutes. Caspase-3 activity was detected by CaspSELECT Caspase-3 Immunoassay kit (MBL International Corp.) after BMSSCs were treated with staurosporin at $1 \mu \mathrm{M}$ for 4 hours in the presence or absence of pretreatment with Z-DEVD-FMK for 24 hours. The osteogenic inductive condition in vitro was as previously reported (44).

Transplantation of BMSSCs into immunocompromised mice. Approximately $4.0 \times 10^{6}$ of mouse or human BMSSCs were mixed with $40 \mathrm{mg}$ of HA/
TCP ceramic powder (Zimmer Inc.) and then transplanted into the dorsal surface of 10-week-old immunocompromised beige mice (bg-nu/nu-xid; Harlan), as previously described (45). The transplants harvested at 8 weeks after transplantation were fixed in $4 \%$ paraformaldehyde and then decalcified with $10 \%$ EDTA ( $\mathrm{pH}$ 8.0) for paraffin embedding. Paraffin sections were deparaffinized, hydrated, and stained with $\mathrm{H} \& \mathrm{E}$. For quantification of new bone regeneration in vivo, NIH Image software (http://rsb.info.nih. gov/nih-image) was used as previously described (43).

Histology. Skeletal histomorphometric parameters were assessed at the distal femoral metaphyses of 3-week-old mice. Bones were dehydrated and embedded in a solution containing 97\% methylmethacrylate (SigmaAldrich), 3\% dibutylphthalate (Sigma-Aldrich), and $0.25 \%$ perkadox (Akzo Nobel). Longitudinal cross-sections ( $4 \mu \mathrm{m}$ in thickness) were stained with Massons trichrome for the measurement of bone volume BV/TV and OS/BS. Bone formation indices, including MAR, MS/BS, and BFR, were measured on the sections labeled with calcein. All measurements were made at $\times 200$ magnification using a semiautomatic digitizing system (Bioquant System 4; R\&M Biometrics Inc.) attached to a microscope with a bright-field light source (Nikon Optihot 2 microscope; Nikon Co.).

Alizarin red and alcian blue staining of mouse skeleton. Casp $3^{---}, \mathrm{Casp}^{+--}$, and WT littermates at 14.5 and 17.5 d.p.c. and 1 week were dissected to remove the skin, muscle, and fat; fixed with ethanol for 4 days; and then preserved in acetone for 3 days to further remove fat. Mice were stained with alizarin red $(0.09 \%)$ and alcian blue $(0.05 \%)$ in a solution containing ethanol, glacial acetic acid, and water (67:5:28) for 3 days. After staining, mouse samples were transferred to $1 \% \mathrm{KOH}$ until their soft tissues were dissolved and then preserved in glycerol solution (46).

Bone analyses by microCT and $\mathrm{PQCT}$. Distal femoral metaphyses were analyzed by $\mu \mathrm{CT}$ ( $\mu \mathrm{CT}-20$; SCANCO USA Inc.). Scanning regions were confined to secondary spongiosa and were approximately $0.30 \mathrm{~mm}$ in thickness. Using 2-dimensional images, a region of interest was manually drawn near the endocortical surface. Cancellous bone morphometric indices, assessed using 3-dimensional image reconstructions, included BV/TV (\%), Tb.Th, trabecular number, and trabecular separation. PQCT analysis of the distal femora was performed using an XCT Research M scanner (Stratec; Norland Co.), as previously described (47). Briefly, scans were obtained at $2.25 \mathrm{~mm}$ and $2.75 \mathrm{~mm}$ from the distal condyles and cancellous BMD. Machine cancellous BMD precision (based on the manufacturer's data) was $\pm 3 \mathrm{mg} / \mathrm{cm}^{3}$, while the coefficient of variation in our laboratory based on repeat scans was $2.26 \%$.

CFU-F assay. The CFU-F assay was performed as previously described (48). Cell aggregates containing more than 50 cells were counted as colonies using a dissecting microscope. The CFU-F assay was repeated in 5 independent experiments.

Population doubling. Mouse BMSSCs were cultured at low density to form single cell-derived colonies and then were trypsinized and seeded at a density of $2-5 \times 10^{5}$ cells in $100-\mathrm{mm}$ culture dishes at the first passage. Upon reaching confluency, the cells were trypsinized and seeded at the same cell density. The population doubling was calculated at every passage according to the equation: $\log _{2}$ (number of harvested cells/number of seeded cells). The finite population doublings were determined by cumulative addition of total numbers generated from each passage until the cells ceased dividing (49).

Histocytochemistry. BMSSCs cultured under the osteogenic inductive condition were fixed with $4 \%$ paraformaldehyde. Calcium deposits were detected by staining with $1 \%$ alizarin red ( $\mathrm{pH} 4.2$; Sigma-Aldrich). The proliferation rate of BMSSCs was assessed by BrdU incorporation for 24 hours using a BrdU Staining Kit (Zymed Laboratories Inc.). For Annexin V staining, mouse BMSSCs from each genotype were seeded on chamber slides and treated with $2 \mathrm{ng} / \mathrm{ml}$ TGF- $\beta$ for 3 days. Then an Annexin-V-FLUOS Staining Kit (Roche Diagnostics Co.) was used to detect apoptotic cells according to the manufacturer's instructions. 
$\beta$-Gal staining. $\beta$-Gal staining was performed as a marker of replicative senescence according to the previously described procedure (50). Briefly, BMSSCs were washed 3 times with PBS and then fixed with $2 \%$ formaldehyde and with $0.2 \%$ glutaraldehyde for 5 minutes. The cells were washed 3 times with PBS and then incubated overnight at $37^{\circ} \mathrm{C}$ in a solution containing $40 \mathrm{mM}$ citrate- $\mathrm{Na}_{2} \mathrm{HPO}_{4}$ buffer ( $\mathrm{pH}$ 6.0), $5 \mathrm{mM}$ potassium ferrocyanide, $5 \mathrm{mM}$ potassium ferricyanide, $150 \mathrm{mM} \mathrm{NaCl}, 2 \mathrm{mM} \mathrm{MgCl} 2$, and $1 \mathrm{mg} / \mathrm{ml} \mathrm{X}$-gal. After incubation, the cells were washed twice with PBS and photographed, and the percentage of stained cells was counted.

Preosteoblast isolation. Preosteoblasts were isolated from calvariae of 1- to 3-day-old Casp $3^{--}$, Casp $3^{+-}$, and WT mice by sequential digestion with 200 $\mathrm{U} / \mathrm{ml}$ of collagenase type I (Worthington Biochemical Corp.). Preosteoblasts were cultured in $\alpha$-MEM supplemented with $10 \% \mathrm{FBS}, 2 \mathrm{mM}$ L-glutamine, and a combination of $100 \mathrm{U} / \mathrm{ml}$ penicillin and $100 \mu \mathrm{g} / \mathrm{ml}$ streptomycin until they became $70 \%$ confluent. The cells were serum starved in a manner similar to BMSSCs upon treatment with $2 \mathrm{ng} / \mathrm{ml}$ TGF- $\beta$.

Osteoclast induction in vitro. The osteoclast induction assay was performed by coculture of nucleated spleen cells with preosteoblasts obtained from calvariae. Preosteoblasts were plated on 48-well plates at a density of $10^{4}$ cells $/ \mathrm{cm}^{2}$ with phenol red-free MEM (Gibco BRL; Invitrogen Corp.) supplemented with $10 \% \mathrm{FBS}$, nonessential amino acids (Mediatech Inc.), $2 \mathrm{mM}$ L-glutamine, and a combination of $100 \mathrm{U} / \mathrm{ml}$ penicillin and $100 \mu \mathrm{g} / \mathrm{ml}$ streptomycin. After preosteoblasts were incubated for 16 hours, nucleated spleen cells $\left(5 \times 10^{5}\right.$ cells $\left./ \mathrm{cm}^{2}\right)$ were added with $100 \mathrm{nM}$ dexamethasone, $50 \mu \mathrm{M}$ ascorbic acid 2-phosphate, and 0.5 or $2 \mathrm{nM} 1,25(\mathrm{OH})_{2} \mathrm{D}_{3}$ (Biomol Research Laboratories Inc.). Spleen cells were harvested from 5 -week-old mice. The medium was changed every 3 days. After 5 days, the cells were stained for TRAP and the number of $\mathrm{TRAP}^{+}$-MNCs was determined as previously described (51).

Osteoclast function in vivo. A murine calvarial osteolysis model was used to estimate inductive bone resorption as previously described (52). Briefly, $5 \times 10^{5}$ titanium particles (Johnson Matthey Public Ltd.) in $40 \mu \mathrm{l}$ PBS were implanted on the surface of parietal bones of each mouse. After 7 days, parietal bones were harvested and processed for X-ray analysis using the Faxitron MX-20 Specimen Radiography System (Faxitron X-ray Co.). The extent of osteolysis in each bone was determined from the X-ray image by using NIH Image software.

1. Strasser, A., O’Connor, L., and Dixit, V.M. 2000 Apoptosis signaling. Annu. Rev. Biochem. 69:217-245.

2. Nicholson, D.W. 1999. Caspase structure, proteolytic substrates, and function during apoptotic cell death Cell Death Differ. 6:1028-1042.

3. Kuida, K., et al. 1996. Decreased apoptosis in the brain and premature lethality in CPP32-deficient mice. Nature. 384:368-372.

4. Woo, M., et al. 1998. Essential contribution of caspase $3 / \mathrm{CPP} 32$ to apoptosis and its associated nuclear changes. Genes Dev. 12:806-819.

5. Kennedy, N.J., Kataoka, T., Tschopp, J., and Budd, R.C. 1999. Caspase activation is required for T cell proliferation. J. Exp. Med. 190:1891-1896.

6. Zermati, Y., et al. 2001. Caspase activation is required for terminal erythroid differentiation. J. Exp. Med. 193:247-254.

7. Fernando, P., Kelly, J.F., Balazsi, K., Slack, R.S., and Megeney, L.A. 2002. Caspase 3 activity is required for skeletal muscle differentiation. Proc. Natl. Acad. Sci. U. S. A. 99:11025-11030.

8. Hock, J.M., et al. 2001. Osteoblast apoptosis and bone turnover. J. Bone Miner. Res. 16:975-984.

9. Manolagas, S.C. 1999. Cell number versus cell vigor-what really matters to a regenerating skeleton? Endocrinology. 140:4377-4381.

10. Most, W., et al. 1995. In vitro and ex vivo evidence that estrogens suppress increased bone resorption induced by ovariectomy or PTH stimulation

Western blot analysis. Cells were lysed in M-PER extraction reagent (Pierce Chemical Co.), and protein concentrations were measured using Bio-Rad Protein Assay (Bio-Rad Laboratories Inc.). Ten micrograms of protein were applied to each lane and separated on Tris-Glycine SDS-PAGE gel (Novex; Invitrogen Corp.). The proteins were then transferred onto BA-S 85 nitrocellulose membranes (Schleicher \& Schuell BioScience Inc.) and blocked for 3 hours at room temperature in NAP-Blocker (Geno Technology Inc.). Antibodies used for Western blot included: rabbit anti-RANKL, HSP 90, TGF- $\beta$ RI, TGF- $\beta$ RII, Cdk2 and Cdc2, and mouse anti-p21 and p53 (Santa Cruz Biotechnology Inc.); rabbit anti-p-Smad2 (Cell Signaling Technology Inc.); rabbit anti-Cbfa1 (Oncogene Research Products); rabbit anti-Smad2 and Smad3 (Zymed Laboratories Inc.). Membranes were incubated with the first antibodies (1:1,000 dilution) for 1 hour at room temperature and then incubated with HRP-conjugated secondary antibodies (Santa Cruz Biotechnology Inc.) at 1:5,000 dilution for 1 hour at room temperature. After they were immunolabeled, membranes were washed and reacted with Super Signal chemiluminescence HRP substrate (Pierce Chemical Co.) and then visualized on Kodak X-Omat film (Eastman Kodak Co.).

Statistical analysis. Student's $t$ test was used to analyze significance between 2 groups. A $P$ value of less than 0.05 was considered significant.

\section{Acknowledgments}

We thank Larry Fisher for providing antibodies and Tina Kilts for animal technical support. This work was supported by the intramural program of NIDCR/NIH, Department of Health and Human Services.

Received for publication October 29, 2003, and accepted in revised form October 19, 2004.

Address correspondence to: Songtao Shi, Building 30, Room 222, NIDCR/NIH, 30 Convent Drive MSC-4320, Bethesda, Maryland 20892, USA. Phone: (301) 435-4584; Fax: (301) 402-0824; E-mail: sshi@dir.nidcr.nih.gov.

Masako Miura and Xiao-Dong Chen contributed equally to this work.

through an effect on osteoclastogenesis. J. Bone Miner. Res. 10:1523-1530.

11. Cenci, S., et al. 2000. Estrogen deficiency induces bone loss by enhancing T-cell production of TNF- $\alpha$. J. Clin. Invest. 106:1229-1237.

12. Jilka, R.L., Weinstein, R.S., Takahashi, K., Parfitt, A.M., and Manolagas, S.C. 1996. Linkage of decreased bone mass with impaired osteoblastogenesis in a murine model of accelerated senescence. J. Clin. Invest. 97:1732-1740.

13. Pavalko, F.M., et al. 2003. Fluid shear stress inhibits TNF-alpha-induced apoptosis in osteoblasts: a role for fluid shear stress-induced activation of PI3 kinase and inhibition of caspase-3. J. Cell. Physiol. 194:194-205.

14. Kim, G.S., et al. 2003. Leptin induces apoptosis via ERK/cPLA2/cytochrome c pathway in human bone marrow stromal cells. J. Biol. Chem. 278:21920-21929.

15. Mogi, M., and Togari, A. 2003. Activation of caspases is required for osteoblastic differentiation. J. Biol. Chem. 278:47477-47482.

16. Hay, E., Lemonnier, J., Fromigue, O., and Marie, P.J. 2001. Bone morphogenetic protein-2 promotes osteoblast apoptosis through a Smad-independent, protein kinase C-dependent signaling pathway. J. Biol. Chem. 276:29028-29036.

17. Adams, C.S., Mansfield, K., Perlot, R.L., and Shapiro, I.M. 2001. Matrix regulation of skeletal cell apoptosis. Role of calcium and phosphate ions. J. Biol. Chem. 276:20316-20322.

18. Plotkin, L.I., et al. 1999. Prevention of osteocyte and osteoblast apoptosis by bisphosphonates and calcitonin. J. Clin. Invest. 104:1363-1374.

19. Kalu, D.N., and Chen, C. 1999. Ovariectomized murine model of postmenopausal calcium malabsorption. J. Bone Miner. Res. 14:593-601.

20. Kasukawa, Y., Stabnov, L., Miyakoshi, N., Baylink, D.J., and Mohan, S. 2002. Insulin-like growth factor I effect on the number of osteoblast progenitors is impaired in ovariectomized mice. J. Bone Miner. Res. 17:1579-1587.

21. Jin, Y.H., Yoo, K.J., Lee, Y.H., and Lee, S.K. 2000. Caspase 3-mediated cleavage of p21WAF1/CIP1 associated with the cyclin A-cyclin-dependent kinase 2 complex is a prerequisite for apoptosis in SK-HEP-1 cells. J. Biol. Chem. 275:30256-30263.

22. Rebbaa, A., Zheng, X., Chou, P.M., and Mirkin, B.L. 2003. Caspase inhibition switches doxorubicin-induced apoptosis to senescence. Oncogene. 22:2805-2811.

23. Massague, J. 1998. TGF--beta signal transduction. Annu. Rev. Biochem. 67:753-791.

24. Whitman, M. 1998. Smads and early developmental signaling by the TGF-beta superfamily. Genes Dev. 12:2445-2462.

25. Law, B.K., et al. 2002. Rapamycin potentiates transforming growth factor beta-induced growth arrest 
in nontransformed, oncogene-transformed, and human cancer cells. Mol. Cell. Biol. 22:8184-8198.

26. Alliston, T., Choy, L., Ducy, P., Karsenty, G., and Derynck, R. 2001. TGF-beta-induced repression of CBFA 1 by Smad 3 decreases cbfa 1 and osteocalcin expression and inhibits osteoblast differentiation. EMBO J. 20:2254-2272.

27. Eblen, S.T., Fautsch, M.P., Burnette, R.J., Joshi, P., and Leof, E.B. 1994. Cell cycle-dependent inhibition of $\mathrm{p} 34 \mathrm{cdc} 2$ synthesis by transforming growth factor beta 1 in cycling epithelial cells. Cell Growth Differ. 5:109-116.

28. Fautsch, M.P., Eblen, S.T., Anders, R.A., Burnette, R.J., and Leof, E.B. 1995. Differential regulation of $\mathrm{p} 34 \mathrm{cdc} 2$ and $\mathrm{p} 33 \mathrm{cdk} 2$ by transforming growth factor-beta 1 in murine mammary epithelial cells. J. Cell. Biochem. 58:517-526.

29. Geng, Y., and Weinberg, R.A. 1993. Transforming growth factor beta effects on expression of G1 cyclins and cyclin-dependent protein kinases. Proc. Natl. Acad. Sci. U. S. A. 90:10315-10319.

30. Ewen, M.E., Sluss, H.K., Whitehouse, L.L., and Livingston, D.M. 1993. TGF- beta inhibition of Cdk4 synthesis is linked to cell cycle arrest. Cell. 74:1009-1020.

31. Polyak, K., et al. 1994. p27Kip1, a cyclin-Cdk inhibitor, links transforming growth factor-beta and contact inhibition to cell cycle arrest. Genes Dev. 8:9-22.

32. Datto, M.B., et al. 1995. Transforming growth factor beta induces the cyclin-dependent kinase inhibitor p21 through a p53-independent mechanism. Proc. Natl. Acad. Sci. U. S. A. 92:5545-5549.

33. McDonnell, T.J., and Korsmeyer, S.J. 1991. Progression from lymphoid hyperplasia to high-grade malignant lymphoma in mice transgenic for the t(14; 18). Nature. 349:254-256.

34. Watanabe-Fukunaga, R., Brannan, C.I., Copeland, N.G., Jenkins, N.A., and Nagata, S. 1992. Lymphoproliferation disorder in mice explained by defects in Fas antigen that mediates apoptosis. Nature. 356:314-317.

35. Thompson, C.B. 1995. Apoptosis in the pathogenesis and treatment of disease. Science. 267:1456-1462.

36. Saikumar, P., et al. 1999. Apoptosis: definition, mechanisms, and relevance to disease. Am. J. Med. 107:489-506

37. Simpson, M.T., et al. 2001. Caspase 3 deficiency rescues peripheral nervous system defect in retinoblastoma nullizygous mice. J. Neurosci. 21:7089-7098.

38. Yoshizawa, K., et al. 2000. Caspase-3 inhibitor rescues $\mathrm{N}$-methyl- $\mathrm{N}$-nitrosourea-induced retinal degeneration in Sprague-Dawley rats. Exp. Eye Res. 71:629-635.

39. Hoglen, N.C., et al. 2001. Characterization of the caspase inhibitor IDN-1965 in a model of apoptosis-associated liver injury. J. Pharmacol. Exp. Ther. 297:811-818.

40. Ruetten, H., Badorff, C., Ihling, C., Zeiher, A.M., and Dimmeler, S. 2001. Inhibition of caspase-3 improves contractile recovery of stunned myocardium, independent of apoptosis-inhibitory effects. J. Am. Coll. Cardiol. 38:2063-2070.

41. Hotchkiss, R.S., et al. 2000. Caspase inhibitors improve survival in sepsis: a critical role of the lymphocyte. Nat. Immunol. 1:496-501.

42. Nicholson, D.W. 2000. From bench to clinic with apoptosis-based therapeutic agents. Nature. 407:810-816

43. Shi, S., et al. 2002. Bone formation by human post- natal bone marrow stromal stem cells is enhanced by telomerase expression. Nat. Biotechnol. 20:587-591.

44. Gronthos, S., Mankani, M., Brahim, J., Robey, P.G., and Shi, S. 2000. Postnatal human dental pulp stem cells (DPSCs) in vitro and in vivo. Proc. Natl. Acad. Sci. U. S. A. 97:13625-13630.

45. Krebsbach, P.H., et al. 1997. Bone formation in vivo: comparison of osteogenesis by transplanted mouse and human marrow stromal fibroblasts. Transplantation. 63:1059-1069.

46. Zhao, M., et al. 2002. Bone morphogenetic protein receptor signaling is necessary for normal murine postnatal bone formation. J. Cell Biol. 157:1049-1060.

47. Nielsen, K.L., et al. 2003 Biglycan deficiency interferes with ovariectomy-induced bone loss. J. Bone Miner. Res. 18:2152-2158.

48. Kuznetsov, S., and Gehron Robey, P. 1996. Species differences in growth requirements for bone marrow stromal fibroblast colony formation in vitro. Calcif. Tissue Int. 59:265-270.

49. Blazer, S., et al. 2002. High glucose-induced replicative senescence: point of no return and effect of telomerase. Biochem. Biophys. Res. Commun. 296:93-101.

50. Dimri, G.P., et al. 1995. A biomarker that identifies senescent human cells in culture and in aging skin in vivo. Proc. Natl. Acad. Sci. U. S. A. 92:9363-9367.

51. Ragab, A.A., Lavish, S.A., Banks, M.A., Goldberg, V.M., and Greenfield, E.M. 1998. Osteoclast differentiation requires ascorbic acid. J. Bone Miner. Res. 13:970-977.

52. Kaar, S.G., et al. 2001. Rapid repair of titanium particle-induced osteolysis is dramatically reduced in aged mice. J. Orthop. Res. 19:171-178. 